\section{Maria Cecília de Souza Minayo \\ Fátima Gonçalves Cavalcante}

\title{
Suicídio entre pessoas idosas: revisão da literatura
}

\section{Suicide in elderly people: a literature review}

\section{RESUMO}

Foi realizada revisão da literatura sobre os principais fatores associados à ideação, tentativas e suicídio propriamente dito de pessoas idosas. Foram pesquisadas as seguintes bases de dados: MEDLINE, PsychInfo, SciELO e Biblioteca Virtual em Violência e Saúde da BIREME, referentes ao período de 1980 a 2008. Foram selecionadas e analisadas 52 referências, que mostraram forte relação entre ideação, tentativas e efetivação do ato fatal em pessoas idosas, que resulta da interação de fatores complexos: físicos, mentais, neurobiológicos e sociais. O suicídio associado à depressão em idosos é prevenível desde que a pessoa esteja devidamente tratada. Há necessidade de investimento em pesquisa no Brasil, dado o crescimento persistente das taxas de suicídio entre idosos, sobretudo do sexo masculino.

DESCRITORES: Idoso. Suicídio. Tentativa de Suicídio. Fatores de Risco. Revisão.

\begin{abstract}
A literature review was carried out focusing on the main factors associated with suicidal ideation, attempts and completed suicide in elders. The following databases were searched: MEDLINE, PsychINFO, SciELO and Biblioteca Virtual em Violência e Saúde da BIREME (BIREME's Violence and Health Virtual Library), referring to the period from 1980 to 2008. Fifty-two references were selected and analyzed. They showed a strong relationship among suicide ideation, attempt and completion in elderly individuals, which results from the interaction of complex physical, mental, neurobiological and social factors. Suicide associated with depression in the elderly can be prevented, provided the person is properly treated. In Brazil, it is necessary to invest in research, given the persistent increase in suicide rates among aged people, especially among males.
\end{abstract}

\author{
Correspondência | Correspondence: \\ Maria Cecília de Souza Minayo \\ Centro Latino-Americano de Estudos sobre \\ Violência e Saúde \\ Av. Brasil, $4036 / 7^{\circ}$ andar \\ Manguinhos \\ 21040-210 Rio de Janeiro, RJ, Brasil \\ E-mail: maminayo@terra.com.br
}

DESCRIPTORS: Aged. Suicide. Suicide, Attempted. Risk Factors. Review. 


\section{INTRODUÇÃO}

Segundo a Organização Mundial da Saúde (OMS) ${ }^{45}$ o suicídio vitima cerca de um milhão de pessoas no mundo por ano. Para a OMS, a violência autodirigida se manifesta de duas formas: no comportamento suicida (por meio de pensamentos, tentativa e pelo suicídio consumado) e por meio de atos violentos provocados contra a própria pessoa, como é o caso das mutilações.

As informações da literatura mostram que as estatísticas de suicídio se distribuem desigualmente pelo mundo, dentro dos países, entre os sexos e entre os grupos de idade. Por exemplo, no caso da dispersão geográfica entre os países, Japão e algumas sociedades européias ganham em relevância: suas taxas se assemelham às de homicídios no Brasil. ${ }^{18,19}$ No mundo como um todo, em números absolutos, os suicídios matam mais que os homicídios e as guerras juntos. No Brasil, as taxas de suicídio são baixas se comparadas à da maioria dos países, oscilando entre 3,50 e 4,00 por 100.000 habitantes, contrastando com as de homicídio, muito mais elevadas..$^{35}$

O suicídio entre pessoas idosas constitui hoje um grave problema para as sociedades das mais diversas partes do mundo. Estudo realizado pelo WHO/Euro Multicentre Study of Suicidal Behaviour ${ }^{18}$ em 13 países europeus mostra que as taxas médias de suicídio entre pessoas com mais de 65 anos nessas sociedades chega a 29,3/100.000 e as de tentativas de suicídio, a $61,4 / 100.000$. Além de os dados sobre autodestruição em idosos serem muito elevados, a razão entre tentativas e suicídios consumados é muito próxima, quase 2:1. Um conjunto de pesquisas, no mesmo sentido, leva a concluir que, quando uma pessoa idosa tenta se matar, há que se levar seu gesto muito a sério, pois é provável que qualquer tentativa redunde no ato de dar cabo à própria vida ${ }^{34,54}$ De Leo et al ${ }^{18}$ ressaltam ainda que, na Europa Ocidental, as tentativas de suicídio entre pessoas idosas diminuem com a idade e, ao inverso, existe o crescimento de suicídio consumados. Esse aumento de suicídios consumados no final da vida também foi mostrado nos EUA, em pesquisa de Nock et al. ${ }^{37}$

A população acima de 60 anos é a que mais cresce no Brasil e na maior parte do mundo, o que justifica um olhar atento para os problemas sociais e de saúde que a afetam. Segundo constatação de Beeston, “o crescimento das taxas de suicídio entre idosos indica que o aumento da idade se relaciona com processos biológicos e psicológicos que podem induzir a pessoa à decisão de se autodestruir". ${ }^{5}$ Essa situação pode ser constatada inclusive no Brasil, onde, apesar de as taxas de suicídio serem relativamente baixas, as que se referem à população na faixa etária acima de 60 anos são o dobro das que a população em geral apresenta, principalmente devido ao aumento crescente das taxas relativas ao grupo de homens idosos..$^{34}$
Embora relevante, o suicídio de pessoas idosas tem merecido pouca atenção, não só no Brasil, mas no mundo inteiro. ${ }^{5,7,16,36,47-49}$ Não foram encontradas referências do Brasil ou países de língua espanhola sobre o suicídio entre idosos na base de dados SciELO, apenas sobre suicídio em geral: 340 referências de 1981 a 2009, sobretudo estudos realizados na última década.

O objetivo do presente estudo foi analisar fatores associados (doença mental, enfermidades físicas e problemas sociais) ao risco de suicídio de pessoas idosas, a partir da literatura indexada nas bases de dados nacionais e internacionais.

\section{MÉTODOS}

Foi realizada uma revisão narrativa e compreensiva de estudos e pesquisas sobre o fenômeno do suicídio em pessoas idosas. Foram também consideradas tentativas e ideação suicida, haja vista a estreita relação entre esses três momentos e movimentos quando se trata desse grupo etário. ${ }^{57}$

Considerou-se a tendência recente de buscar a força da evidência em diferentes fontes, abordagens e metodologias, especialmente em países em desenvolvimento. Estudos clínicos e randomizados, pautados na força da validade interna ou na efetividade e evidenciados pelos dados quantitativos, não devem ser a única fonte para assegurar a qualidade ou o rigor de uma revisão de literatura, especialmente quando se abordam temas menos explorados na saúde pública. . $^{39,56}$

Foram selecionados estudos que problematizaram a assistência ao idoso na área da psiquiatria, neurologia, enfermagem e psicologia, bem como das ciências sociais. Áreas mais recentes, como a neurociência, neuropsiquiatria, neuropsicologia, psicogeriatria e psicologia médica, agregaram novas especificidades que enriqueceram a noção de prevenção, diagnóstico, tratamento, evolução e manejo dos fatores associados ao suicídio em idosos ou das condições de vida e de adoecimento mental ou físico. ${ }^{52}$ Além disso, foram revistos estudos clínicos de caso controle, autópsias psicológicas e estudos multicêntricos sobre o fenômeno.

As referências apresentadas pela literatura sobre suicídio em pessoas idosas foram coletadas a partir das bases de dados MEDLINE, PsychINFO, SciELO, Biblioteca Virtual em Violência e Saúde da Bireme e o livro de Chesnais, ${ }^{10}$ que estudou o comportamento do fenômeno por 200 anos na Europa. Foram analisados também estudos que investigaram a difusão e o impacto do suicídio para o setor saúde. ${ }^{56,57}$

As palavras-chaves utilizadas na busca revisão foram: "suicide and elderly"; "suicide and older people"; 
"suicide and aged people"; "suicide and suicide attempts and older people". Pesquisaram-se também os termos em português: "suicídio entre pessoas idosas" e em espanhol: "suicidio de personas mayores". O estudo abrangeu publicações partir dos anos 1980 até 2008. A base de maior relevância para o trabalho foi MEDLINE, seguida da PsychINFO. Não foram encontradas pesquisas na base SciELO. Foram analisadas 57 referências, sendo 52 sobre o tema proposto; as cinco restantes referem metodologia para estudos de suicídio e procedimentos da modalidade de revisão narrativa.

As referências selecionadas foram analisadas pelas tendências das últimas três décadas. Entre 1980 e 1989 observou-se que o tema era incipiente: foram encontrados cinco artigos. De 1990 a 1999, os estudos quadruplicaram. Houve 19 trabalhos cujos eixos temáticos são o suicídio, a co-morbidade com transtornos mentais, o diagnóstico e o tratamento. Na última década, de $2000 \mathrm{a}$ 2009, foram selecionadas 28 referências, observando-se crescimento de quantidade e diversidade das pesquisas sobre o assunto. Os eixos temáticos principais são suicídio, depressão e prevenção; comportamento suicida, transtornos mentais e físicos e condições de vida. Pela diversidade de procedimentos e métodos investigativos não foi possível hierarquizar evidências pelo rigor com que o conceito é tratado hoje. No entanto, buscou-se assinalar a força de categorias recorrentes a partir de diferentes fontes.

Os dados foram sistematizados em cinco categorias: 1) visão sobre a literatura das últimas décadas; 2) relações entre ideação, tentativas e suicídios consumados entre pessoas idosas e relação do suicídio com 3) doenças mentais, 4) enfermidades físicas e 5) fatores sociais.

\section{A LITERATURA NAS ÚLTIMAS DÉCADAS}

Em 52 referências, 90\% dizia respeito a trabalhos desenvolvidos nos últimos 20 anos. Na década de 1990 encontram-se estudos a partir de autópsias psicológicas e descrições clínicas, visando compreender pensamentos suicidas, situar fatores predisponentes e incrementar a avaliação e o tratamento do idoso em risco de suicídio. A partir de 1995, as investigações se ampliam e aparecem estudos de caso controle, de prevalência e co-morbidade com enfermidade mental. Enfoques sobre o desejo de morrer em idosos acima de 85 anos e sobre o prognóstico de idosos com depressão e risco de suicídio já começaram a ser destacados nesse período.

A década de 2000 é acompanhada de um volume substancial de trabalhos. Ampliam-se pesquisas de caso controle, desenvolvem-se estudos multicêntricos nacionais e internacionais, e há grande investimento em pesquisas estratégicas e baseadas em evidências, buscando reunir rigor técnico e relevância social. ${ }^{46} \mathrm{O}$ termo "comportamento suicida" aparece em trabalhos de enfoque clínico e epidemiológico. ${ }^{18,37}$ Ampliam-se estudos sobre depressão, suicídio e envelhecimento. A atenção primária e os cuidados em saúde mental passam a integrar formas de prevenção do suicídio associado a depressão e outras enfermidades psiquiátricas. Estudos mais específicos enfatizam traços de personalidade, fatores associados a doenças físicas, estresse psicossocial e suas implicações médicas, psiquiátricas e neuropsicológicas.

\section{RELAÇÃO ENTRE SUICÍDIO, TENTATIVAS E IDEAÇÃO}

Revisão realizada por Beeston ${ }^{5}$ indica consenso entre os estudiosos a respeito da intrínseca relação entre ideação, tentativa e efetivação do ato suicida. ${ }^{21,24,27,29,39,50}$ Forsell et $\mathrm{l}^{21}$ mostram relação entre ideação e depressão e entre ideação e dependências múltiplas, institucionalização, severos problemas visuais e uso de drogas psicotrópicas. Qualquer investigação sobre o tema da ideação é problemática, uma vez que esse fenômeno é pouco perguntado por pesquisadores e relatado pelos idosos; quando isso ocorre, pode ser confundido com processos depressivos. Os pesquisadores dizem que muitos deles se referem a "pensamentos de morte", "desejos de morrer", "cansaço de viver", "falta de sentido da vida" e "tristeza com o rumo atual da própria existência".

Em relação às tentativas, existem dados mais concretos. Por exemplo, estudos analisados por Beeston ${ }^{5}$ mostram que nos Estados Unidos existe a relação de 36:1 entre tentativas e efetivação de suicídios de jovens, de 8:1 na população em geral; entre idosos, essa relação é muito estreita: $4: 1$. Hawton et $a^{24}$ também identificaram que o hábito de se machucar ou as tentativas de suicídio devem ser considerados fortes preditores de eventual suicídio na população idosa, ressaltando a pequena diferença entre o número dos que tentam e dos que consumam o ato. Todos os artigos contemplados e que tratam da questão de gênero ressaltam que as mulheres idosas pensam mais em suicídio e os homens estão na frente na efetivação do suicídio.

Osgood \& Thielmann ${ }^{38}$ consideram que a relação entre ideação, tentativas e efetivação do suicídio pode ser encontrada em comunicações verbais, comportamentos, casos situacionais e alguns conjuntos de sinais. No caso da manifestação verbal, a pessoa idosa pode ir diretamente ao assunto, confidenciando a pessoas próximas ou respondendo a alguém que a agride que quer morrer. Muitas vezes, porém, a pessoa idosa apenas insinua seu desejo de morte, comentando sobre isso com familiares, amigos e companheiros.

No caso de comportamentos sintomáticos, o mais previsível é a própria tentativa. Segundo estudiosos, há várias condutas que deveriam ser consideradas verdadeiros alertas aos cuidadores e parentes: descuido com 
a medicação, colocação em ordem dos pertences ou dos haveres, desinteresse pelas coisas da vida, busca súbita de alguma religião ou igreja, desinteresse em se cuidar, freqüentes visitas ao médico com sintomas vagos. ${ }^{13} \mathrm{Em}$ alguns países, como os EUA, a compra de arma por um homem idoso deve ser considerado forte sinal de que ele esteja pensando em suicídio. ${ }^{8}$

Fatores situacionais são todos os eventos que estejam acontecendo na vida de uma pessoa mais idosa e que lhe provoquem depressão, melancolia e tristeza. Alguns podem ser mencionados como sendo os mais comuns na literatura analisada: o status de aposentado quando tem como conseqüência despir o idoso de sua função social, refugiá-lo em casa ou isolá-lo socialmente; a morte de um dos cônjuges, filhos(as) ou amigos; o diagnóstico de uma doença grave; a perda das referências sociais, como a privação de espaço na própria casa.

Entre os fatores sindrômicos, Beeston ${ }^{5}$ e Holkup ${ }^{26}$ descreveram um conjunto de sintomas: depressão acompanhada de ansiedade; tensão, agitação, sentimento de culpa e dependência de outrem; rigidez, impulsividade e isolamento; mudanças nos hábitos de comer e de dormir; súbita recuperação em relação a um quadro de depressão profunda.

\section{RELAÇÃO ENTRE DOENÇA MENTAL E SUICÍDIO}

A literatura mostra que doenças e transtornos mentais estão fortemente relacionados com suicídios em pessoas idosas. As autópsias psicológicas-estudos que reúnem informações post mortem a respeito das circunstâncias e situações do suicídio de uma pessoa, com a finalidade de compreender as razões que a motivaram a cometer o ato fatal - reportam que entre $71 \%$ e $95 \%$ das pessoas idosas que cometeram suicídio possuíam diagnóstico de algum transtorno mental por ocasião de sua morte. ${ }^{2-4-}$ ,9,12-14,22,25,53,55 Harwood et al $^{22}$ encontraram numa amostra bastante significativa de idosos que se suicidaram que $77 \%$ deles padeciam de algum distúrbio psiquiátrico quando cometeram o ato, dos quais $63 \%$ sofria de depressão e $44 \%$ apresentava algum outro problema, como rigidez na forma de ver a vida e obsessão. Por outro lado, Rao et $\mathrm{al}^{40}$ não encontraram associação de suicídio com enfermidades como Alzheimer, demências severas e outras enfermidades ligada à memória.

Com efeito, o suicídio será sempre um evento complexo e de causas múltiplas. ${ }^{35}$ No entanto, vários pesquisadores mostram que transtornos afetivos e principalmente depressão sejam sintomas associados. ${ }^{28,30}$ Bruce at al, ${ }^{8}$ em estudo realizado com a população americana acima de 65 anos, observaram que a depressão profunda estaria presente em cerca de $1 \%$ a $2 \%$ deles e $10 \%$ a $15 \%$ padeciam de sintomas depressivos menos graves. No caso dos idosos, ela freqüentemente associa o processo de adoecimento mental a problemas de saúde física, ao isolamento e à falta de suporte social, fatores esses que também se relacionam à ocorrência de suicídio. As principais expressões da depressão são: persistente mau humor, falta de interesse e de alegria de viver, sentimento de que as energias estão se esvaindo, tristeza, atitudes negativas, fadiga constante e persistente, distúrbios do sono e do apetite, desesperança e vontade de sumir ou de morrer. ${ }^{30,43}$

Para Beautrais et al, ${ }^{3}$ a forte associação entre suicídio e depressão leva a recomendar sérios cuidados em relação aos que apresentam esse sofrimento mental. Beeston $^{5}$ confirma, a partir de extensa revisão de várias pesquisas, ${ }^{10,41-43}$ que o tratamento e o manejo da depressão talvez seja o fator singular mais importante na prevenção de suicídio em idosos. Embora exista um preconceito segundo o qual as pessoas idosas sejam naturalmente deprimidas por causa da idade, a depressão não é um fato normal do envelhecimento. ${ }^{5}$

\section{RELAÇÃO ENTRE ENFERMIDADES FÍSICAS E SUICÍDIO}

A presença de algumas doenças graves é considerada um fator de risco para o suicídio de pessoas idosas. Alguns estudos ${ }^{10,23,32}$ mostram que essa associação é mais significativa para as seguintes enfermidades: câncer, alguns problemas no sistema nervoso central, complicações cardiopulmonares e doenças urogenitais em homens. Entretanto, pesquisadores consideram que a experiência de uma enfermidade física grave pode provocar depressão em idosos (considerada fator desencadeante), mas não existe relação direta entre o estado de saúde física e ideação ou tentativa de suicídio. ${ }^{5}$

Numerosos estudos, porém, vêm examinando a relação entre ideação e tentativas suicidas e o enfrentamento de enfermidades terminais. Brown et $\mathrm{al}^{6}$ encontraram uma em cada quatro pessoas que expressaram o desejo de acabar com a vida, dentre 44 doentes terminais idosos. Desse total, também $25 \%$ tinham diagnóstico de depressão. A relação entre enfermidade terminal e suicídio foi confirmada por estudos de Conwell et al. ${ }^{17}$ Contudo, tais autores concluem que os problemas mentais, sobretudo a depressão - que geralmente ocorre também associada ao estado de grande sofrimento físico - são os maiores fatores de risco. ${ }^{12}$ Pesquisas de Waern et al, ${ }^{55}$ Conwell et $\mathrm{al}^{12,13}$ e Ahearn et $\mathrm{al}^{1}$ mostram que a associação entre risco para suicídio e enfermidades graves em pessoas idosas existe sobretudo para os homens.

Recentemente, trabalhos na área de neurobiologia têm investigado possíveis associações entre distúrbios neurológicos e suicídio. Mann et $\mathrm{al}^{33}$ consideram que baixos níveis de seratonina podem estar associados a comportamentos agressivos e impulsivos de pacientes deprimidos que apresentam evidência de tristeza, 
desesperança e ideação suicida. Outras pesquisas, no mesmo sentido, foram realizadas por Ahearn et $\mathrm{al}^{1} \mathrm{e}$ King et al. ${ }^{28}$ Em geral, os estudos neurobiológicos não são conclusivos, e os autores que entraram nessa linha de pesquisa ressaltam a necessidade de maior aprofundamento de hipóteses recentes. Da mesma forma, a relação entre enfermidades físicas e suicídio é referida com mais propriedade apenas no caso de doenças terminais. A precaução de realizar mais estudos é recomendada fortemente por Beeston:" "Existe uma clara necessidade de mais pesquisas que possam esclarecer a relação entre mudanças neurobiológicas e suicídio. É preciso desenvolver tecnologias nessa área para que tenhamos um melhor entendimento da neuroquímica do suicídio, levando ao desenvolvimento de novas terapias".

\section{RELAÇÕES ENTRE FATORES SOCIAIS E SUICÍDIO}

Várias pesquisas mostram que existem diferenças significativas sobre os fatores sociais de risco para suicídio que afetam pessoas jovens, adultas e idosas. Suicídio pode acontecer em qualquer família e em qualquer grupo social. Entretanto, jovens e adultos que tentam ou cometem suicídio são impelidos por problemas interpessoais (sobretudo amorosos), financeiros, legais ou de desempenho escolar ou no trabalho.

Já para as pessoas idosas, tais fatores são principalmente: morte de uma pessoa querida, mormente de um cônjuge; doença terminal com dores incontroláveis; medo do prolongamento da vida sem dignidade, trazendo prejuízos econômicos e emocionais aos familiares; isolamento social; mudanças nos papéis sociais que lhes conferiam reconhecimento; ou situações de dependência física ou mental diante das quais o idoso se sente humilhado.

Estudos por meio de autópsias sociopsicológicas ${ }^{12,20,44}$ têm reportado que problemas financeiros, dificuldades de relacionamento, brigas na família, isolamento social e solidão ${ }^{18,25}$ são os motivos sociais mais freqüentes de desencadeamento de suicídios em idosos. Alguns autores distinguem entre o viver sozinho - o que pode ser uma opção do idoso - e a solidão. A solidão pode ocorrer com o idoso convivendo com muitas pessoas. A situação de isolamento social e de solidão afeta principalmente os homens, ${ }^{9,20}$ tornando-se, no caso deles, um fator de risco para suicídio. Ao contrário, o cultivo de amizades e de relacionamentos é um fator protetivo importante tanto contra a depressão quanto contra a ideação e efetivação da autodestruição. Autores como Beautrais, ${ }^{4}$ ressaltando a importância do contato humano e do suporte social para que os idosos não coloquem em risco sua vida, reconhecem que a falta de interação social é um dos mais relevantes problemas a serem enfrentados na prevenção do suicídio nesse grupo social, mesmo no caso em que as pessoas não sofram transtornos mentais. Esse último ponto é reforçado pelas pesquisas de Duberstein et al..$^{20}$ Segundo Beeston, ${ }^{5}$ "um sentido de conectividade social e de participação na vida comunitária parece ser protetiva contra o suicídio em pessoas idosas. ${ }^{31,51}$ Portanto, o desenvolvimento de uma rede social de suporte para essas pessoas deveria ser definido como prioridade. Mas devemos alertar que a rede de relações pessoais e de amizade das pessoas idosas vem sendo cada vez mais substituída por serviços profissionais pagos. Isso pode ser problemático para eles".

\section{CONSIDERAÇÕES FINAIS}

As informações apresentadas dizem respeito à realidade de outras sociedades, uma vez que pesquisas sobre o tema, no Brasil, não foram encontradas nas bases de dados, ainda que seja nesse grupo que as taxas de eventos fatais por suicídio mais cresceram nos últimos anos no País.

Considera-se, no entanto, que a apreciação dos textos inova ao introduzir essa discussão no meio acadêmico e no âmbito das políticas e práticas em saúde, além de contribuir para levantar hipóteses a serem testadas em estudos de abrangência nacional e local. A situação que o quadro brasileiro mostra vai ao encontro dos achados internacionais, o que tem levado a OMS a considerar o suicídio a partir de 65 anos como um dos mais sérios problemas atuais de saúde pública. ${ }^{29}$

A análise dos trabalhos apresenta algumas questões: (1) a complexidade do fenômeno, que inclui contribuição de problemas físicos, neurobiológicos, psicológicos e sociais. A maioria dos estudos indica como fatores predisponentes: doenças graves e degenerativas, dependência física, distúrbios e sofrimentos mentais e, sobretudo, depressão severa. (2) A depressão é o mais relevante fator associado ao suicídio, na quase totalidade das pesquisas. Essa enfermidade é vista em sua sintomatologia própria ou associada a questões de sofrimento físico crônico e de terminalidade da vida ou, ainda, a problemas de ordem social e cultural, como perdas, abandonos, solidão ou conflitos no interior das famílias..$^{11,12-17}$ (3) Diferenças de gênero devem ser consideradas. Condizente com todo o conhecimento universal sobre suicídio em qualquer idade, as mulheres idosas têm mais ideação e produzem mais tentativas de dar cabo à vida; os homens são os que mais chegam ao ato final. (4) Numa gradação etária entre idosos, o grupo acima de 80 anos é o que mais se envolve com pensamentos, sentimentos, tentativas e execução do suicídio.

Do ponto de vista do papel da saúde pública, existe uma relação próxima entre ideação, tentativas e ato fatal na população idosa, de tal forma que as manifestações do desejo de se matar ou alguma ação nesse sentido devem ser tratadas imediatamente, buscando as causas mais 
próximas e evitando-se a consumação do ato, haja vista a literatura mostrar que é possível prevenir o suicídio atuando nos fatores associados. Do ponto de vista dos cuidados médicos, é preciso focalizar a depressão como o fator singular desencadeador mais relevante.
Outras ações devem ser promovidas, como os cuidados médicos que ajudem a diminuir sofrimentos e dependências, e ações sociais que auxiliem pessoas idosas a terem uma vida em interação com sua comunidade e a chegarem com dignidade ao final da vida. 


\section{REFERÊNCIAS}

1. Ahearn EP, Jamison KR, Steffens DC, Cassidy $R$, Provenzale JM, Lehman A, et al. MRI correlates of suicide attempts history in unipolar depression. Biol Psychiatry. 2001;50(4):266-70. DOI:10.1016/S00063223(01)01098-8

2. Barclough MB. Suicide in the elderly: recent developments in psycho geriatrics. Br I Psychiatry Suppl. 1971;6(Special):87-97.

3. Beautrais AL, Joyce PR, Mulder RT, Fergusson. DM, Deavoll BJ, Nightingale SK. Prevalence and comorbidity of mental disorders in person making serious attempts: a case control study. Am J Psychiatry. 1996;153(8):1009-14.

4. Beautrais AL. A case-control study of suicide and attempted suicide in older adults. Suicide Life Threat Behav. 2002;32(1):1-9. DOI:10.1521/suli.32.1.1.22184.

5. Beeston D. Older people and suicide. Stoke on Trent: Centre for Ageing and Mental/Health Staffordshire University; 2006.

6. Brown $\mathrm{JH}$, Heteleff $\mathrm{P}$, Barakat $\mathrm{S}$, Rowe $\mathrm{CJ}$. It is normal for terminally ill patients to desire death? Am J Psychiatry.1986;143(2):208-11.

7. Bruce ML. Designing and intervention to prevent suicide. Dialogues Clin Neurosci. 1999;1:100-10.

8. Bruce ML, Ten Have TR, Reynolds 3rd CF, Katz II, Schulberg HC, Mulsant BH, et al. Reducing suicidal ideation and depressive symptoms in depressed older primary care patients: a randomized controlled trial. JAMA. 2004;291(9):1081-91. DOI:10.1001/ jama.291.9.1081

9. Carney SS, Rich CL, Burke PA, Fowler RC. Suicide over 60: the San Diego study. I Am Geriatr Soc. 1984;42(2):174-80.

10. Chesnais JC. Histoire de la violence en occident de 1800 à nos jours. Paris: Robert Laffont; 1981.

11. Cole MG, Bellavance F, Mansour A. Prognosis of depression in elderly community and primare care populations: a systematic review and meta-analysis. Am J Psychiatry. 1999;156(8):1182-9.

12. Conwell Y, Olsen K, Caine ED, Flannery C. Suicide in later life: psychological autopsy findings. Int Psychogeriatr. 1991;3(1):59-66. DOI:10.1017/ S1041610291000522

13. Conwell Y, Brent D. Suicide and aging. I: patterns of psychiatric diagnosis. Int Psychogeriatr. 1995;7(2):14964. DOI:10.1017/S1041610295001943

14. Conwell Y, Duberstein PR, Cox C, Herrmann JH, Forbes NT, Caine ED. Relationship of age and axis I diagnoses in victims of completed suicide: a psychological autopsy study. Am J Psychiatry. 1996;153(8):1001-8.

15. Conwell Y, Lyness GM, Duberstein P, Cox C, Seidlitz L, DiGiorgio A, et al. Completed suicide among older patients in primary care practices: a controlled study. J Am Geriatr Soc. 2000;48(1):23-9.

16. Conwell Y, Duberstein PR, Caine ED. Risk factors for suicide in later life. Biol Psychiatry. 2002;52(3):193204. DOI:10.1016/S0006-3223(02)01347-1
17. Conwell Y, Thompson C. Suicidal behavior in elders. Psichiatr Clin North Am. 2008;3(2)1:333-56.

18. De Leo D, Padoani W, Scocco P, Lie D, et al. Attempted and completed suicide in older subjects: results from the WHO/EURO Multicentre study of suicidal behaviour. Int J Geriatr Psychiatr. 2001;16(3):300-10. DOI:10.1002/gps.337

19. Doak MW, Nixon AC, Lupton DJ, Warning WS. Selfpoisoning in older adults: patterns of drug ingestion and clinical outcomes. Age Ageing. 2009;38(4):40711. DOI:10.1093/AGEING/AFP046

20. Duberstein PR, Conwell Y, Conner KR, Eberly S, Caine ED. Suicide at 50 years of age and older: perceived physical illness, family discord and financial strain. Psychol Med. 2004;34(1):137-46. DOI:10.1017/ S0033291703008584

21. Forsell $Y$, Jorm AF, Winblad B. Suicidal thoughts and associated factor in an elderly population. Acta Psychiatr Scand. 1997;95(2):108-11. DOI:10.1111/ j.1600-0447.1997.tb00382.x

22. Hardwood D, Hawton K, Hope T, Jacoby R. Psychiatric disorders and personality factors associated with suicide in older people: a descriptive and case-control study. Int J Geriatr Psychiatr. 2001;16(2):155-65. DOI:10.1002/1099-1166(200102)16:2<155::AIDGPS289>3.0.CO;2-0

23. Harris EC, Barraclough BM. Suicide as an outcome for medical disorders. Medicine (Baltimore). 1994;73(6):281-96.

24. Hawton K, Appleby L, Platt S, Foster T, Cooper J, Malmberg A, et al. The psychological autopsy approach to studying suicide: a review of methodological issues. J Affect Disord. 1998;50(2-3):269-76.

25. Henriksson MM, Marttunen MJ, Isometsä ET, Heikkinen ME, Aro HM, Kuoppasalmi KI, et al. Mental disorders in elderly suicide. Int Psychogeriatr. 1995;7(2):275-86. DOI:10.1017/S1041610295002031

26 Holkup P. Evidence-based protocol elderly suicide: secondary prevention. J Gerontol Nurs. 2003;29(6):617.

27. Jorm AF, Henderson AS, Scott R, Korten AE, Christensen H, Mackinnon AJ. Factor associated with the wish to die in elderly people. Age Aging.1995;24(5):389-392. DOI:10.1093/ ageing/24.5.389

28. King DA, Conwell Y, Cox C, Henderson RE, Denning DG, Caine ED. A neuropsychological comparison of depressed suicide attempters and nonattempters. I Neuropsychiatry Clin Neurosci. 2000;12(1):64-70.

29. Krug EG, Dahlberg LL, Mercy JA, Zwi AB, Lozano R, editors. World report on violence and health. Geneva: World Health Organization; 2002. Self-directed violence; p.183-210.

30. Linden M, Barrow S. IPA/Bayer Research Awards in Psychogeriatrics. The wish to die in very old persons near the end of life: a psychiatric problem? Results from the Berlin aging study. Int Psychogeriatr. 1997;9(3):291307. DOI:10.1017/S1041610297004456 
31. Lliffe $S$, Manthorpe J. The prevention of suicide in later life: a task for GPs?Br J Gen Pract. 2005;55(513):269273.

32. Mackenzie TB, Popkin MK. Suicide in the medical patient. Int J Psychiatry Med. 1987;17(1):3-22.

33. Mann JJ, Waternaux C, Haas GL, Malone KM. Toward a clinical model of suicidal behaviour in psychiatric patients. Am J Psychiatry. 1999;156(2):181-9.

34. Minayo MCS, Cavalcante FG, Souza ER. Methodological proposal for studying suicide as a complex phenomenon. Cad Saude Publica. 2006;22(8):1587-96. DOI:10.1590/S0102$311 \times 2006000800007$

35. Minayo MCS. Suicídio de pessoas idosas e fatores associados a esse fenômeno no Brasil e no mundo. In: Berzins MV, Malagutti W, organizadores. Rompendo o silêncio: faces da violência na velhice. São Paulo: Martinari; 2009. p.199-218.

36. Mitty E, Flores S. Suicide in late life. Geriatr Nurs. 2009;29(3):160-5. DOI:10.1016/j. gerinurse.2008.02.009

37. Nock MK, Borges G, Bromet EJ, Cha CB, Kessler RC, Lee S. Suicide and suicide behavior. Epidemiol Rev. 2008;30:133-54. DOI:10.1093/epirev/mxn002

38. Osgood NJ, Thielman S. Geriatric suicidal behaviour: assessment and treatement of suicidal patients. In: Blumenthal SJ, Kupter DJ, editors. Suicide over the life cycle: risk factors, assessment and treatment of suicidal patients. Washington: American Psychiatric Press; 1990. p.341-79

39. Petticrew M. Systematic reviews in public health: old chestnuts and new challenges. Bull World Health Organ. 2009;87:163-163A. DOI:10.2471/ BLT.09.063719

40. Rao R, Dening T, Brayne C, Huppert FA. Suicidal thinking in community residents over eighty. Int J Geriatr Psychiatry. 1997;12(3):337-43. DOI:10.1002/ (SICI)1099-1166(199703)12:3<337::AIDGPS498>3.0.CO;2-T

41. Reinolds 3rd CF, Frank E, Perel JM, Imber S, Cornes C, Miller MD, et al. Nortriptyline and interpersonal psychotherapy as maintenance therapies for recurrent Mjor depression. JAMA. 1999;281(1):39-45. DOI:10.1001/jama.281.1.39

42. Reynolds 3rd CF, Dew MA, Pollock BG, Mulsant BH, Frank E, Miller MD, et al. Maintenance treatment of major depression in old age. New Engl / Med. 2006;354(11):1130-8. DOI:10.1056/NEJMoa052619

43. Rosenstein LD. Differential diagnosis of the major progressive dementias and depression in middle and latte adulthood: a summary of the literature of the early 1990s. Neuropsychol Rev. 1998;8(3):109-67. DOI:10.1023/A:1025628925796

44. Rubenowitz E, Waern M, Wilhelmson K, Allebeck P. Life events and psychosocial factors in elederly suicides
- a case control study. Psychol Med. 2001;31(7):1193202. DOI:10.1017/S0033291701004457

45. Saz P, Dewey ME. Depression, depressive symptoms and mortality in persons aged 65 and over living in the community: a systematic review of the literature. Int J Geriatr Psychiatry. 2001;16(6):622-30. DOI:10.1002/ gps.396

46. Segone M, editor. Country-led monitoring and evaluation systems: better evidence, better polices, better development results. Geneva: UNICEF, 2009.

47. Shah A, Bhat $R$. The relationship between elderly suicide rates and mental health funding, service provision and national policy: a cross-national study. Int Psychogeriatr. 2008;20(3):605-15. DOI:10.1017/ S1041610207006552

48. Shah A, Bhat R. Are elderly suicide rates improved by increased provision of mental health service resources? A cross-national study. Int Psychogeriatr. 2008;20(6):1230-7. DOI:10.1017/S1041610208007023

49. Shah A, Padayatchi M, Das K. The relationship between elderly suicide rates and elderly dependency ratios: a cross-national study using data from the WHO data bank. Int Psychogeriatr. 2008;20(3):596-604. DOI:10.1017/S104161020700628X

50. Skoog I, Aevarsson O, Beskow J, Larsson L, Palsson $\mathrm{S}$, Waern $\mathrm{M}$, et al. Suicidal feelings in a population sample of nondemented 85-years-old. Am J Psychiatry.1996;153(8):1015-20.

51. Tadros G, Salib E. Elderly suicide in primary care. Int J Geriatr Psychiatry. 2007;22(8):750-6. DOI:10.1002/ gps. 1734

52. UK Clinical Research Collaboration. Health research classification system. London; 2009.

53. Useda JD, Duberstein PR, Conner KR, Conwell Y. Personality and attempted suicide in depressed adults 50 years of age and older: a facet level analysis. Compr Psychiatry. 2004;45(5):353-61. DOI:10.1016/j. comppsych.2004.06.002

54. Vannoy SD, Duberstein P, Cukrowicz K, Lin E, Fan $M Y$, Unützer J. The relationship between suicide ideation and late-life depression. Am / Geriatr Psychiatry. 2007;15(12):1024-33. DOI:10.1097/ JGP.0b013e3180cc2bf1

55. Waern M, Runeson B, Allebeck P, Beskow J, Rubenowitz E, Skoog I, et al. Mental disordes in elderly people. Am J Psychiatry. 2002;159(3):450-5. DOI:10.1176/appi.ajp.159.3.450

56. Waters E. Evidence for public health decision-making towards reliable synthesis. Bull World Health Organ. 2009;87(3):164. DOI:10.2471/BLT.09.064022

57. Williams VL, Eiseman E, Landree E, Adamson DM. Demonstrating and communicating research impact: preparing NIOSH Programs for External Review. Santa Monica: RAND Corporation; 2009. 